\title{
A Generative Adversarial Model for Radar Echo Extrapolation Based on Convolutional Recurrent Units
}

Kun Zheng ${ }^{1}$, Yan Liu ${ }^{1}$, Jinbiao Zhang ${ }^{2}$, Cong Luo ${ }^{3}$, Siyu Tang ${ }^{3}$, Huihua Ruan², Qiya Tan ${ }^{1}$, Yunlei Yi ${ }^{4}$, Xiutao $\operatorname{Ran}^{4}$

$5{ }^{1}$ School of Geography and Information Engineering, China University of Geosciences, Wuhan 430074, China

${ }^{2}$ Guangdong Meteorological Observation Data center, Guangzhou 510080, China

${ }^{3}$ GuangDong Meteorological Observatory, GuangDong, 510080, China

${ }^{4}$ Wuhan Zhaotu Technology Co. Ltd., Wuhan 430074, China

Correspondence to: Kun Zheng (ZhengK@cug.edu.cn); Yan_Liu(liuyan_@cug.edu.cn)

10 Abstract. Precipitation nowcasting play a vital role in preventing meteorological disasters and doppler radar data acts as an important input for nowcasting models. The traditional extrapolation method is difficult to model highly nonlinear echo movements. The key challenge of the nowcasting mission lies in achieving high-precision radar echo extrapolation. In recent years, machine learning has made a great progress in the extrapolation of weather radar echoes. However, most of models neglect the multi-modal characteristics of radar echo data, resulting in blurred and unrealistic prediction images. This paper aims to solve this problem by utilizing the feature of the GAN that can enhance the multi-modal distribution modelling, and design the radar echo extrapolation model of GAN-argcPredNet v1.0. The model composed of argcPredNet generator and a convolutional neural network discriminator. In generator, a gate control data memory and output are designed in the rgcLSTM prediction unit of the generator, thereby reducing the loss of spatiotemporal information. In discriminator, model uses a dualchannel input method, which enables it to strictly score according to the true echo distribution, and has a more powerful discrimination ability. Through experiments on the radar data set of Shenzhen, China, the results show that the radar echo hit rate (POD) and critical success index (CSI) increased by $5.5 \%$ and $\% 10.4 \%$ compared with rgcPredNet, the false alarm rate (FAR) is reduced by $15 \% \sim 20 \%$. From the comparison of the result graph and the evaluation index, we also found a problem. The recursive prediction method will produce the phenomenon that the prediction result will gradually deviate from the true value over time. In addition, the accuracy of high-intensity echo extrapolation is relatively low. This is a question worthy of further investigation. In the future, we will continue to conduct research from these two directions.

\section{Introduction}

Precipitation nowcasting refers to the prediction and analysis of rainfall in the target area in a short period of time (0-6 hours) (Bihlo et al. 2019). The important data needed for this work comes from doppler weather radar with high temporal and spatial resolution (Wang et al. 2007). Relevant departments can issue early warning information through accurate nowcasting to avoid loss of economic life. However, this task is extremely challenging due to its very low tolerance to time and position errors (Sun et al. 2014).

The existing nowcasting systems mainly include two types, numerical weather prediction (NWP) and based on radar echo extrapolation (Chen et al. 2020). The widely used optical flow method has problems such as poor capture of fast echo change regions, high complexity of the algorithm and low efficiency (Shang et al. 2017). Since echo extrapolation can be considered as a time series image prediction problem, these shortcomings of optical flow method are expected to be solved by recurrent neural network (RNN) (Giles et al. 1994).

With the continuous development of deep learning, more and more neural networks have been applied to the field of nowcasting. Forecast models such as ConvLSTM and EBGAN-Forecaster show that its extrapolation effect is better than that of optical flow method (Shi et al. 2015, Chen et al.2019). However, these models still have the problem of blurred and unrealistic prediction images (Tian et al. 2020, Xie et al. 2020, Jing et al. 2019). One of the main reasons is that they ignore 
the multi-modal characteristics of radar echo data, using the mean square error and mean absolute error as the loss function, which prefers to model unimodal distribution.

The paper proposes a GAN-argcPredNet network model, which aims to solve this problem through GAN's ability to strengthen the characteristics of multi-modal data modelling. The generator adopts the same deep coding-decoding method as PredNet to establish a prediction model, and uses a new structure of convolutional LSTM as a predictive neuron, which can effectively reduce the loss of spatiotemporal information compared with rgcLSTM. The deep convolutional network is used as the discriminator to classify, and the dual-channel input mechanism is used to strictly judge the distribution of real radar echo images. Finally, based on the weather radar echo data set, the generator and the discriminator are alternately trained to make the extrapolated radar echo map more real and precise.

\section{Relate work}

\subsection{Sequence prediction networks}

The essence of radar echo image extrapolation is the problem of sequence image prediction, which can be solved by implementing an end-to-end sequence learning method (Shi et al. 2015, Sutskever et al.2014). ConvLSTM introduces a convolution operation in the conversion of the internal data state of the LSTM, effectively utilizing the spatial information of

55 the radar echo data (Shi et al. 2015). However, because the location-invariant of the convolutional recursive structure is inconsistent with the natural change motion, TraijGRU was further proposed (Shi et al. 2017). Similarly, ConvGRU introduces convolution operations inside the GRU to enhance the sparse connectivity of the model unit and is used to learn video spatiotemporal features (Ballas et.al 2015). The RainNet network learns the movement and evolution of radar echo based on the U-NET convolutional network for extrapolation prediction (Ayzel et al. 2020). PredNet is based on a deep coding

60 framework and adds error units to each network layer, which can transmit error signals like the human brain structure (Lotter et.al 2016). In order to increase the depth of the network and the connections between modules, Skip-PredNet further introduces skip connections and uses ConvGRU as the core prediction unit. Experiments show that its effect is better than the TraijGRU benchmark (Sato et.al 2018). Although these networks can achieve echo prediction, they have the problem of blurring and unrealistic extrapolated images.

\section{2.2 GAN-based radar echo extrapolation}

The Generative Adversarial Network (GAN) consists of two parts: a generator and a discriminator (Goodfellow et al. 2014). GAN can be an effective model for generating images. Using an additional GAN loss, model can better achieve multi-modal data modelling, and each of its outputs is clearer and more realistic (Lotter et.al 2016). Multiple complementary feature learning strategies show that generative adversarial training can maintain the sharpness of future frames and solve the problem of lack

70 of clarity in prediction (Michael et.al 2015). In this regard, the extrapolators built a generative adversarial network to solve the problem of extrapolated image blur, trying to use this adversarial training to extrapolate more detailed radar echo maps (Singh et al. 2017). Similarly, adversarial network with ConvGRU as the core was proposed, mainly to solve the problem of ConvGRU's inability to achieve multi-modal data modelling (Tian et.al 2020). There are also researchers based on the idea of a four-level pyramid convolution structure, and proposed four pairs of models to generate an adversarial network for radar

75 echo prediction (Chen et al. 2019). It should be noted that the traditional GAN network has the problem of unstable training, which will cause the model unable to learn. Therefore, the design of the nowcasting model should be based on a stable and optimized GAN network. 


\section{Model}

\subsection{Model overview}

80 Radar echo extrapolation refers to the prediction of the dissipation and distribution of future echoes based on the existing radar echo sequence diagram. If the problem is formulated, then each echo maps can be regarded as a tensor $x \in R^{W \times H \times C}, \mathrm{~W} 、 \mathrm{H}$ 、 $\mathrm{C}$ represent the width, height, and number of channels, respectively, and $\mathrm{R}$ represents observing the feature area. If input $\mathrm{M}$ sequence echo maps, predict the $\mathrm{N}$ most likely changes in the future, this problem can be expressed in Eq. (1). This article sets the input sequence $\mathrm{M}$ and output sequence $\mathrm{N}$ to 5 and 7 , respectively.

$85 \hat{x}^{t+1}, \ldots, \hat{x}^{t+N}=\underset{x^{t+1}}{\operatorname{argmax}}, \ldots, x^{t+N} p\left(x^{t+1}, \ldots, x^{t+N} \mid x^{t-M+1}, \ldots, x^{t}\right)$

Unlike other forecasting models, GAN-argcPredNet uses WGAN-gp as a predictive framework. The model solves the problem of training instability through gradient penalty measures (Gulrajani et al.2017). Our model mainly includes two parts: generator and discriminator. As shown in Fig.1, the generator is composed of argcPredNet, which is responsible for learning the potential features of the data and simulating the data distribution to generate prediction samples. Then, the predicted samples and the real samples are input into the discriminator to make a judgment, the real data is judged to be true, and the predicted data is judged to be false. Finally, use Adam to optimize the adversarial loss and then update the parameters of the discriminator, optimize the loss function of the generator once every 5 updates, and complete the update of the generator parameters.

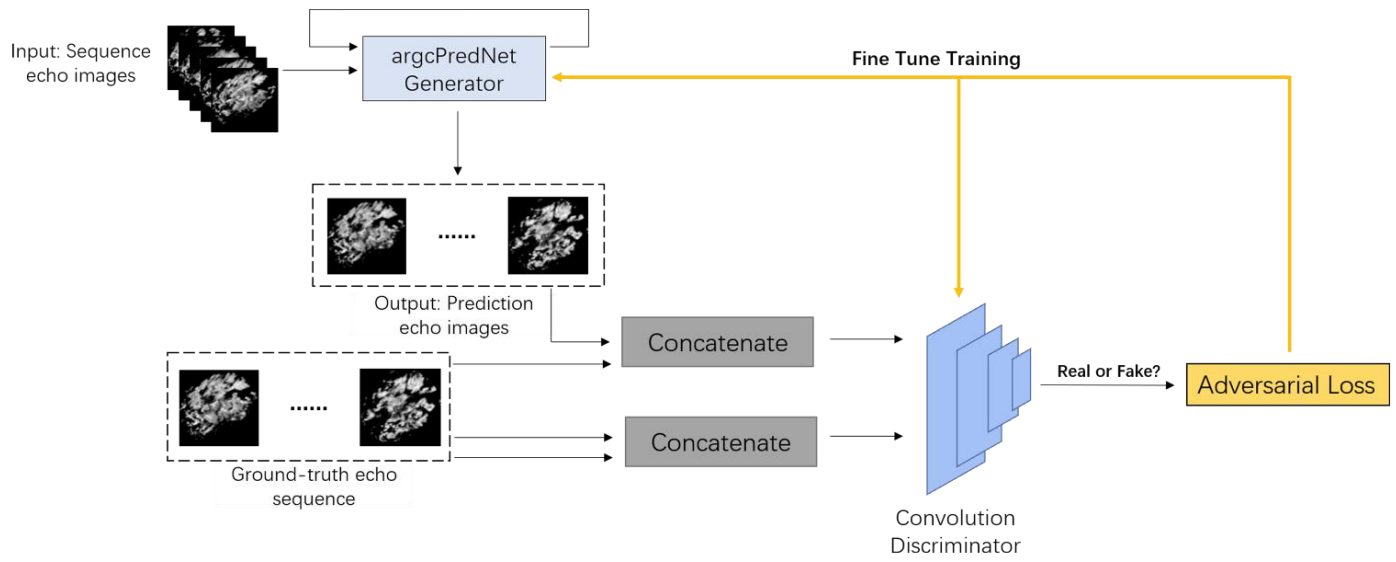

95 Figure 1. Model overview

\section{$3.2 \operatorname{argcPredNet}$ generator}

\subsection{1 $\operatorname{argcLSTM}$}

The internal structure of the argcLSTM neuron used in the model is shown in Fig.2. In order to provide better feature extraction capabilities, the structure contains two trainable gating units, one is the forget gate $f^{(t)}$, the other is the input gate $g^{(t)}$. The latter can calculate the weight of the current state independently, and complete the feature retention of the input information. The peephole connection from the unit state to the forget gate is removed. This operation does not have a big impact on the result, but simplifies the redundant parameters. The complete definition of the argcLSTM unit is as follows (Eq. (2) - Eq. (6)).

$$
\begin{aligned}
& \mathrm{f}^{(\mathrm{t})}=\sigma\left(\mathrm{W}_{\mathrm{fx}} * \mathrm{x}^{(\mathrm{t})}+\mathrm{U}_{\mathrm{fh}} * \mathrm{~h}^{(\mathrm{t}-1)}+\mathrm{b}_{\mathrm{f}}\right) \\
& \mathrm{g}^{(\mathrm{t})}=\sigma\left(\mathrm{W}_{\mathrm{gx}} * \mathrm{x}^{(\mathrm{t})}+\mathrm{U}_{\mathrm{gh}} * \mathrm{~h}^{(\mathrm{t}-1)}+\mathrm{b}_{\mathrm{g}}\right)
\end{aligned}
$$


$\mathrm{C}^{(\mathrm{t})}=\mathrm{f}^{(\mathrm{t})} \circ \mathrm{C}^{(\mathrm{t}-1)}+\mathrm{g}^{(\mathrm{t})} \circ \tilde{\mathrm{C}}^{(\mathrm{t})}$

$\mathrm{h}^{(\mathrm{t})}=\mathrm{g}^{(\mathrm{t})} \circ \tanh \left(\mathrm{C}^{(\mathrm{t})}\right)$

Among them, *represents convolution operation, orepresents Hadamard product, $\sigma$ represents sigmoid nonlinear activation function, $f^{(t)} 、 g^{(t)}$ represent forget gate and update gate, $x^{(t)} 、 h^{(t)} 、 C^{(t)}$ represent the input, hidden state and unit state at time $t$, respectively.

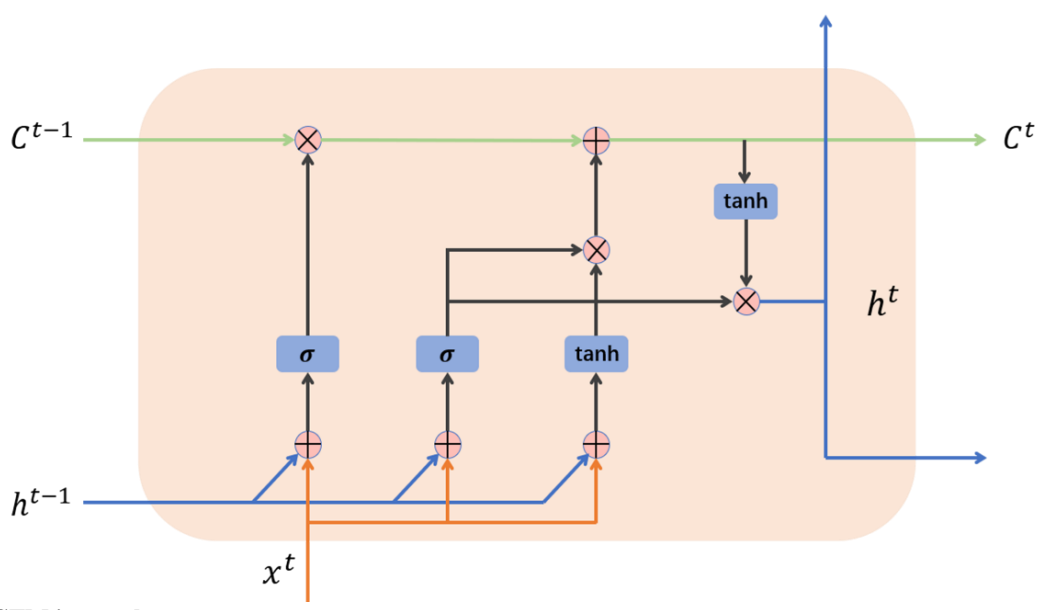

Figure 2. argcLSTM internal structure

\subsection{2 argcPredNet}

The argcPredNet generator has the same structure as PredNet, which is composed of a series of repeatedly stacked modules, stacking a total of 3 layers. The difference is that $\operatorname{argcPredNet}$ uses $\operatorname{argcLSTM}$ as the prediction unit. As shown in Fig 3, each layer of the module contains four units, namely: $A_{l}$ : input convolutional layer, $R_{l}$ : recurrent representation layer, $\hat{A}_{l}$ : prediction convolutional layer, $E_{l}$ : error representation layer.

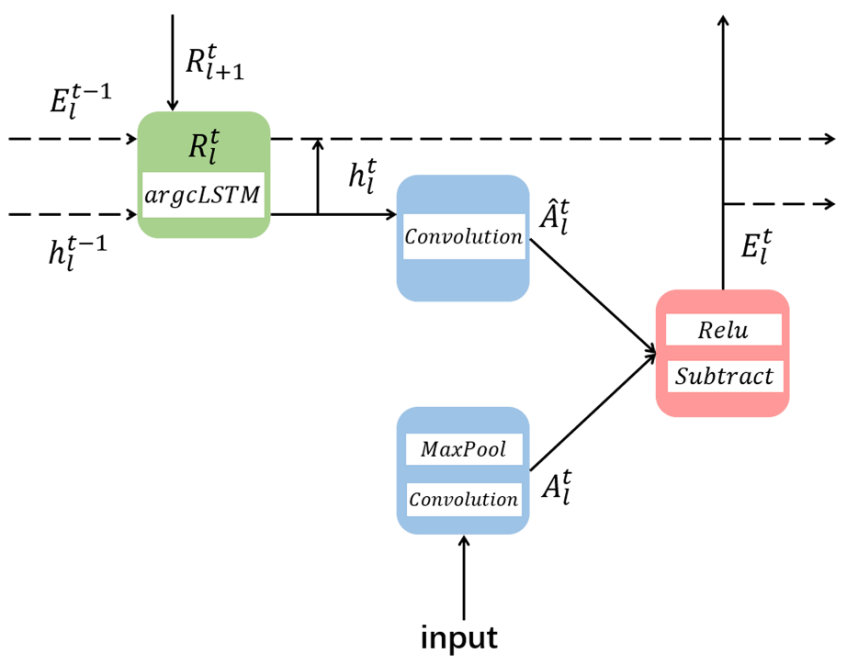

Figure 3. Module expansion diagram of layer $l$ at time $t$ 

the input of $\hat{A}_{l}$ and $A_{l+1}$. The network uses error calculation, $E_{l}$ will output an error representation, and then the error representation is passed forward through the convolutional layer to become the input of the next layer $A_{l+1}$. The hidden state of the recurrent unit $R_{l}^{t}$ is updated according to the output of $E_{l}^{t-1}, R_{l}^{t-1}$ and the up-sampled $R_{l+1}^{t}$. For $A_{l}$, the input of the lowest target, namely $A_{0}$, is set to the actual sequence itself, when $l>0$, the input of $A_{l}$ is: lower error signal $E_{l-1}$ results from convolution calculation, RELU activation and maximum pooling layer. The complete update rules are shown in Eq. (7) to Eq. (10).

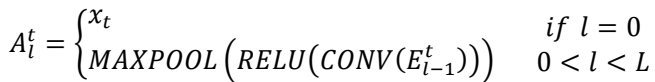

$\hat{A}_{l}^{t}=\operatorname{RELU}\left(\operatorname{CONV}\left(R_{l}^{t}\right)\right)$

$E_{l}^{t}=\left[\operatorname{RELU}\left(A_{l}^{t}-\hat{A}_{l}^{t}\right) ; \operatorname{RELU}\left(\hat{A}_{l}^{t}-A_{l}^{t}\right)\right]$

$R_{l}^{t}= \begin{cases}\operatorname{argcLSTM}\left(E_{l}^{t-1}, R_{l}^{t-1}\right) & \text { if } l=L \\ \operatorname{argcLSTM}\left(E_{l}^{t-1}, R_{l}^{t-1}, \operatorname{UPSAMPLE}\left(R_{l+1}^{t}\right)\right) & 0<l<L\end{cases}$

\subsection{Discriminator and loss}

\subsubsection{Convolutional discriminator}

The purpose of the discriminator is to recognize images, which is similar in nature to the classifier. In the GAN-argcPredNet model, a DC-CNN network is designed for discrimination. The process is shown in Fig.4. It is a four-layer convolution model with a dual-channel input method.

The DC-CNN network extracts a pair of images from the three pairs of images, and inputs them to the fully connected layer through a four-layer convolution transformation, and finally generates a probability output through the Sigmoid function, indicating the possibility that the input image is from a real image. When the input is a real image, the discriminator will maximize the probability, and the value will approach 1. If the input is a generator synthesized image, the discriminator will minimize the probability, and the value will approach -1 .

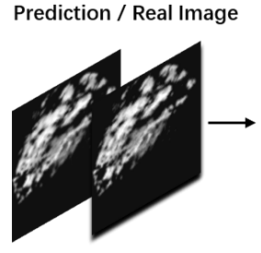

Conv 1 Conv 2 Conv 3 Conv 4

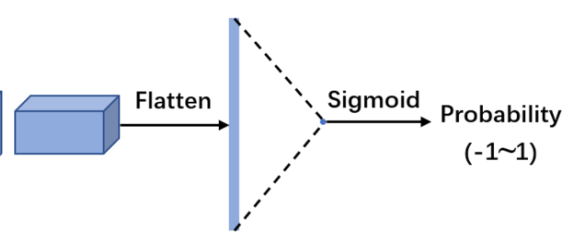

Dense

Figure 4. DC-CNN structure

\subsubsection{Loss function}

The generative adversarial network relies on the distribution of simulated data to generate images. It can retain more echo details, thereby realizing the modelling of multi-modal data. A gradient penalty term is added to GAN-argcPredNet, the loss function of the discriminator is shown in Eq. (11).

$L_{D}=D(\tilde{x})-D(x)+\lambda\left(\left\|\nabla_{\hat{x}} D(\hat{x})\right\|_{2}-1\right)^{2}$

The generator has the following loss function (Eq. (12)).

$L_{G}=E_{\tilde{x} \sim P_{g}}[D(\tilde{x})]-E_{x \sim P_{r}}[D(x)]$ 
Where $\tilde{x}$ represents the distribution of generated samples. $P_{g}$ represents the set of generated sample distributions. $x$ represents the distribution of real samples. $P_{r}$ represents the set of real sample distributions. The third term is the penalty item of the gradient penalty mechanism. In the penalty term, $\hat{x}$ represents the actual data and generation a new sample formed by random sampling between data. $P_{\hat{x}}$ represents a set of randomly sampled samples. $\lambda$ is a hyperparameter, which represents the coefficient of the penalty term, and the value in the model is set to 10 .

\section{Experiments}

In order to verify the effectiveness of the model, the paper uses the radar echo data from January to July 2020 in Shenzhen, China, to conduct experiments on the three models of rgcPredNet, argcPredNet and GAN-argcPredNet. All experiments are implemented in Python, based on the Keras deep learning library, with Tensorflow as the backend for model training and testing.

\subsection{Dataset description}

This experiment uses the radar echo data of Shenzhen China. The data set is all rain images after quality control. The reflectivity range is $0-80 \mathrm{dBZ}$, the amplitude limit is between 0 and 255, and it is collected every 6 minutes, with a total of 1 layer. The height of sea level is $3 \mathrm{~km}$. A total of 600,000 echo images were collected, of which 550,000 were used as the training set and 50,000 were used as the test set for testing. Each set of data contained 12 consecutive images. The horizontal resolution of the radar echo maps is 0.01 degrees (about $1 \mathrm{~km}$ ), the number of grids is $501 * 501$ (that is, an area of about $500 \mathrm{~km} \times 500 \mathrm{~km}$ ), and the image resolution is $96 * 96$ pixels.

\subsection{Evaluation metrics}

170 In order to evaluate the accuracy of the model on precipitation nowcasting, the experiment uses three evaluation indicators to evaluate the prediction precision of the model, critical success index (Eq. (14)), namely false alarm rate (Eq. (15)) and hit rate (Eq. (16)).

$$
\begin{aligned}
C S I & =\frac{T P}{T P+F N+F P} \\
F A R & =\frac{F N}{T P+F N} \\
175 P O D & =\frac{T P}{T P+F P}
\end{aligned}
$$

In the formula, TP indicates that both the predicted value and the true value reach the specified threshold, FN means that the true value reaches the specified threshold but the predicted value has not reached, FP indicates that the true value has not reached the specified threshold but the predicted value has reached the specified threshold.

\subsection{Results}

180 The experiment comprehensively evaluates the prediction accuracy of precipitation with different thresholds. The radar reflectivity and rainfall intensity refer to the Z-R relationship (Shi et al. 2017). The calculation formula is as Eq. (17).

$\mathrm{Z}=10 \log \mathrm{a}+10 \mathrm{~b} \log \mathrm{R}$ 
In this paper, $a$ is set to 58.53 , and $b$ is set to $1.56, \mathrm{Z}$ represents the intensity of radar reflectivity, $R$ represents the intensity of rainfall, and the corresponding relationship between rainfall and rainfall level refers to Table 1 (Shi et al .2017).

185

\begin{tabular}{lc} 
& Table 1. Rain level \\
\hline Rain Rate $\left(\mathrm{mm} \mathrm{h}^{-1}\right)$ & Rainfall Level \\
\hline $0 \leq R<0.5$ & No / Hardly noticeable \\
$0.5 \leq R<2$ & Light \\
$2 \leq R<5$ & Light to moderate \\
$5 \leq R<10$ & Moderate \\
$10 \leq R<30$ & Moderate to heavy \\
$30 \leq R$ & Rainstorm warning \\
\hline
\end{tabular}

Figure 5, Figure 6 and Figure 7 compare the CSI, POD, and FAR index scores of each model at different rainfall thresholds in detail.

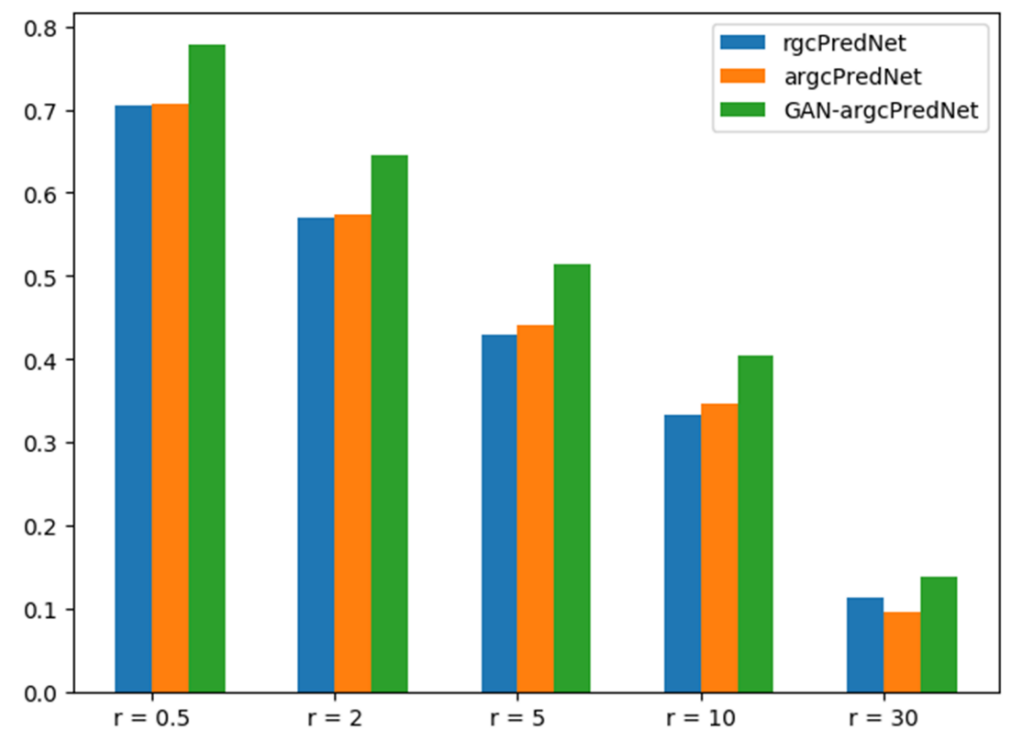

Figure 5.CSI Index score 


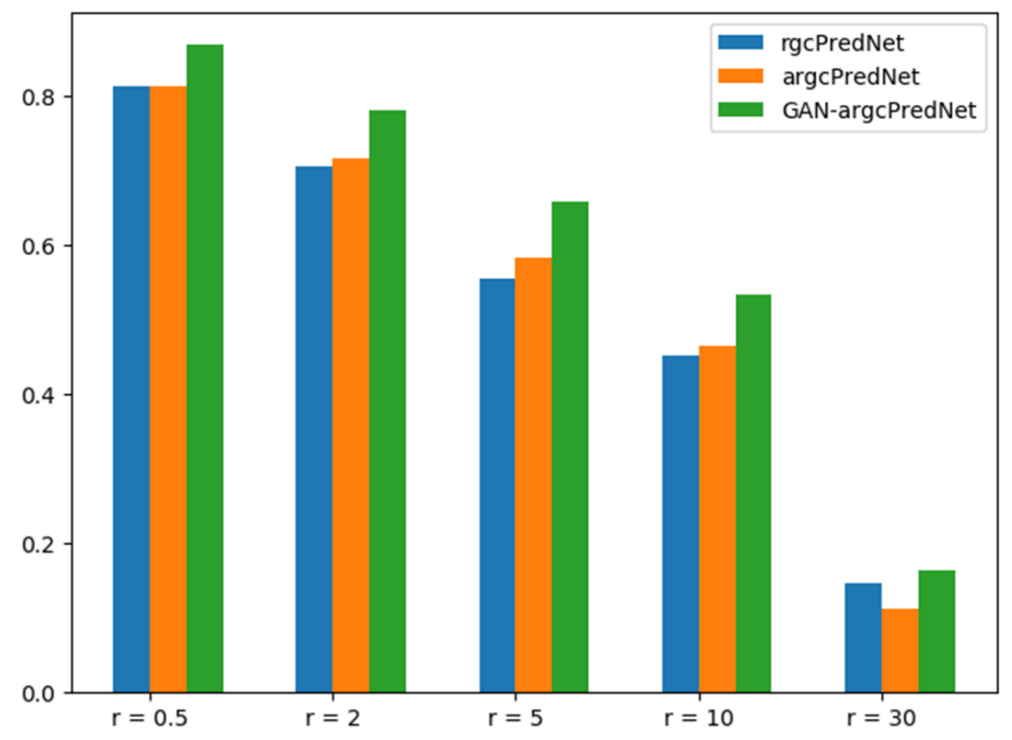

Figure 6. POD Index score

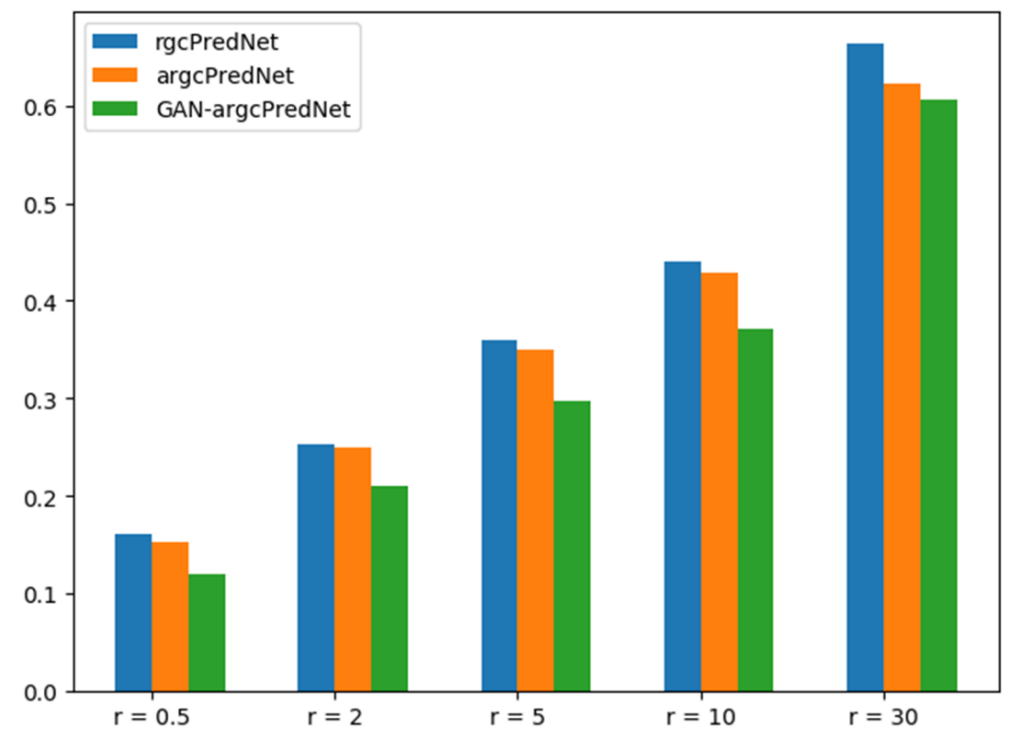

Figure 7. FAR Index score

This result is calculated based on 50,000 test pictures (approximately more than 4,000 test sets), which is representative. It can be seen that when the rainfall rate increases from $0.5 \mathrm{~mm} \mathrm{~h}^{-1}$ to $30 \mathrm{~mm} h^{-1}$, GAN-argcPredNet always performs best, and its advantage is very significant, argcPredNet is the second, and the benchmark model is the worst. Another point worth noting is that as the rainfall intensity increases, the performance of all models shows a downward trend. In the comparison of CSI indicators, GAN-argcPredNet is much ahead of argcPredNet and the benchmark model when the rainfall rate is lower than $30 \mathrm{~mm} \mathrm{~h} h^{-1}$. The performance of the argcPredNet model is not so impressive, and when the Rainfall Level reaches the rainstorm warning, its CSI index is not as good as the benchmark model. For the POD index, the three models have the biggest difference in performance when the rainfall rate is $5 \mathrm{mmh}$. For FAR, the score of $\operatorname{argcPredNet}$ in the interval of $0.5 \mathrm{~mm} h^{-1}$ $10 \mathrm{~mm} h^{-1}$ rainfall rate is slightly lower than that of GAN-argcPredNet, showing relatively good performance. 
To compare the three methods more intuitively, Figure 8 show the image prediction results of the three models on the same piece of test data.

\begin{tabular}{|c|c|c|c|c|c|c|c|}
\hline & $t+1$ & $t+2$ & $t+3$ & $t+4$ & $\mathbf{t}+\mathbf{5}$ & $t+6$ & $t+7$ \\
\hline Ground-truth & & & & & & & \\
\hline
\end{tabular}

rgc_PredNet

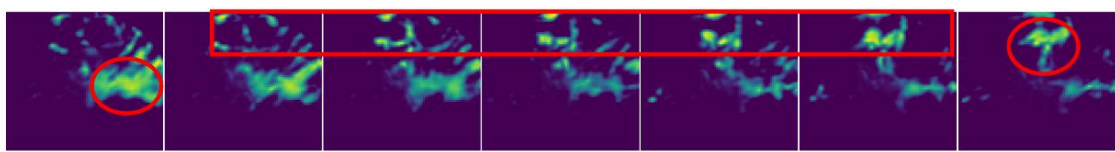

arge_PredNet

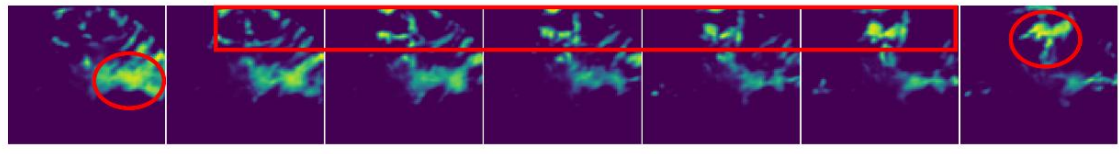

GAN-argcPredNet

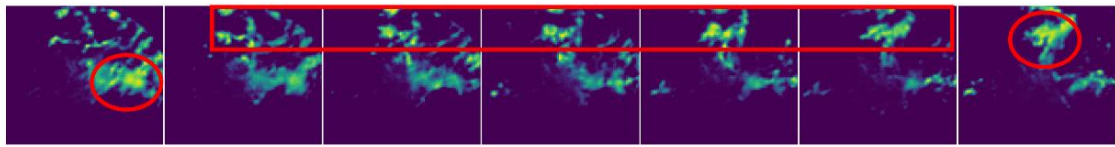

Figure 8. Three prediction examples for the precipitation nowcasting problem. From top to bottom: ground truth frames; prediction by rgcPredNet, prediction by argcPredNet; prediction by GAN-argcPredNet.

Compared with the other three models, GAN-ArgcPredNet generates better image clarity and shows more detailed features on a small scale. The contrast between the areas marked by the red ellipse in Fig. 8 is more obvious. GAN-ArgcPredNet has 210 made the best prediction on the shape and intensity of the echo. The area selected by the rectangle mainly shows the echo changes in the northern region within 30 minutes. Both models correctly predict the movement of the echo to a certain extent, and the prediction process shown by GAN-ArgcPredNe is the most complete. In some mixed intensity and edge areas, our model clearly predicts the echo intensity information, which can be seen the effect of confrontation training is obvious.

In order to compare the prediction results more specifically, the experiment uses Mean Square Error (MSE) and Mean

215 Structural Similarity (MSSIM) to evaluate the quality of the generated images (Wang et al. 2004). The MSE and MSSIM index scores of the images generated by each model are shown in Table 2. Although the MSE index of rgcPredNet is slightly lower than that of the $\operatorname{argcPredNet}$ and GAN-argcPredNet models, the MSSIM index of the argcPredNet and GAN-argcPredNet models is 0.058 and 0.125 higher than that of the rgcPredNet network model, respectively.

Table 2. MSE and MSSIM index scores of each model

\begin{tabular}{ccc}
\hline \multicolumn{3}{c}{ Table 2. MSE and MSSIM index scores of each model } \\
\hline Name & MSE $\times 10^{2} \downarrow$ & MSSIM $\uparrow$ \\
\hline rgcPredNet & 0.496 & 0.732 \\
argcPredNet & 0.476 & 0.790 \\
GAN-argcPredNet & $\mathbf{0 . 4 5 1}$ & $\mathbf{0 . 8 5 7}$ \\
\hline
\end{tabular}

\section{Conclusion}

The study demonstrated a radar echo extrapolation model. The main innovations are summarized as follows. First, the argcPredNet generator is established based on the time and space characteristics of radar data. argcPredNet can predict future echo changes based on historical echo observations. Second, our model uses adversarial training methods to try to solve the problem of blurry predictions. 

can reduce the prediction loss in a small-scale space, so that the prediction results have more detailed features. However, the recursive extrapolation method causes the error to accumulate as time goes by, and the prediction result deviates more and more from the true value. In addition, when the amount of high-intensity echo data is small, the prediction of high-risk and strong convective weather through machine learning is also a problem that we are very concerned about, because it is more realistic. So, we will carry out research on these two issues in the future.

Code and data availability. The GAN-argcPredNet and $\operatorname{argcPredNet}$ models are free and open source. The current version number is GAN-argcPredNet v1.0, and the source code is provided through a GitHub repository https://github.com/LukaDoncic0/GAN-argcPredNet or accessed through the zendo repository https://doi.org/10.5281/zenodo.5035201. The pretrained GAN-argcPredNet and argcPredNet weights are available at https://doi.org/10.5281/zenodo.4765575. The radar data used in the article comes from the Guangdong Meteorological Department. Due to the confidentiality policy, the data will not be disclosed to the public. If you need access to data, please contact Kun Zheng (ZhengK@cug.edu.cn) and Yan Liu (liuyan_@cug.edu.cn).

Author contributions. Kun Zheng was responsible for developing models and writing manuscripts; Yan Liu and Qiya Tan conducted model experiment and co-authored the manuscript; Jinbiao Zhang, Cong Luo, Siyu Tang Huihua Ruan, Yunlei Yi and Xiutao Ran were responsible for data screening and preprocessing.

Competing interests. The authors declare that they have no conflict of interest.

Acknowledgements. This research was funded by Science and Technology Planning Project of Guangdong Province, China (No.2018B020207012).

\section{Reference}

Ayzel, G., Scheffer, T., and Heistermann, M.: RainNet v1.0: a convolutional neural network for radar-based precipitation nowcasting, 13(6):2631-2644, Geoscientific Model Development, https://doi.org/10.5194/gmd-2020-30, 2020.

Ballas, N., Yao L., Pal C., and Courville A.: Delving Deeper into Convolutional Networks for Learning Video Representations, Computer Science, https://arxiv.org/abs/1511.06432, 2015.

Bihlo, A.: Precipitation nowcasting using a stochastic variational frame predictor with learned prior distribution, Computer 250 Science, https://arxiv.org/abs/1905.05037, 2019.

Chen, L., Cao, Y., Ma L., and Jun, Z.: A Deep Learning based Methodology for Precipitation Nowcasting With Radar, Earth and Space Science, 7, 2, https://doi.org/10.1029/2019EA000812, 2020.

Chen, Y.Z., Lin, L. X., Wang, R. Lan, H.P., Ye, Y.M, and Chen, X.L.: A study on the artificial intelligence nowcasting based on generative adversarial networks, Trans Atmos Sci, 42(2), 311- 320, 2019. 

Neural Networks, 5(2), 153-156, 1994.

Goodfellow, I.J., Pouget-Abadie, J., Mirza, M., Xu, B., and Warde-Farley, D., Sherjil ,O., Aaron, C.,and Yoshua, B.: Generative Adversarial Networks, in: Advances in neural information processing systems, 3, 2672-2680, https://arxiv.org/abs/1406.2661, 2014.

260 Gulrajani, I., Ahmed, F., Arjovsky, M., Dumoulin, V., Courville A.: Improved Training of Wasserstein GANs, Computer Science, https://arxiv.org/abs/1704.00028, 2017.

Jing, J., Li, Q., and Peng, X.: MLC-LSTM: Exploiting the Spatiotemporal Correlation between Multi-Level Weather Radar Echoes for Echo Sequence Extrapolation, Sensors, 19(18), 3988, https:/www.mdpi.com/1424-8220/19/18/3988, 2019.

Lotter, W., Kreiman, G., and Cox, D.: Deep predictive coding networks for video prediction and unsupervised learning,

265 Computer Science, https://arxiv.org/abs/1605.08104v5, 2016.

Michael, M., Camille, C., and Yann, L.: Deep multi-scale video prediction beyond mean square error, Computer Science https://arxiv.org/abs/1712.09867v3, 2015.

Sato, R., Kashima, H., and Yamamoto, T.: Short-term precipitation prediction with skip-connected prednet, in: International Conference on Artificial Neural Networks, 373-382, https://doi.org/10.1007/978-3-030-01424-7_37, 2018.

270 Shangzan, G., Da, X., and Xingyuan, Y.: A Short-Term Rainfall Prediction Method Based on Neural Networks and Model Ensemble, Advances in Meteorological Science and Technology, 2017.

Shi, X., Chen, Z., Wang, H., Dit-Yan, Y., Wai-Kin, W., Wang-chun, W.: Convolutional LSTM network: A machine learning approach for precipitation nowcasting, in: Advances in neural information processing systems 28 , edited by Cortes, C.,Lawrence, N. D., Lee, D. D., Sugiyama, M., and Garnett, R., pp. 802-810, https://arxiv.org/abs/1506.04214, 2015.

275 Shi, X., Gao, Z., Lausen, L., Wang, H., Dit-Yan, Y., Wai-Kin, W., Wang-chun, W.: Deep learning for precipitation nowcasting: A benchmark and a new model, in: Advances in neural information processing systems30, edited by Guyon, I., Luxburg, U. V ., Bengio, S., Wallach, H., Fergus, R., Vishwanathan, S., and Garnett, R., pp.5617-5627, https://arxiv.org/abs/1706.03458v1, 2017.

Singh, S., Sarkar, S., Mitra, P.: A deep learning based approach with adversarial regularization for Doppler weather radar echo prediction, 2017 IEEE International Geoscience and Remote Sensing Symposium (IGARSS), 5205-5208, https://ieeexplore.ieee.org/document/8128174, 2017.

Sun, J., Xue, M., Wilson, J. W., ISztar, Z., Sue, P., Jeanette O., Paul, J., Dale, M., Ping-Wah, L., Brian, G., Mei, X., and James, P.: Use of NWP for nowcasting convective precipitation: Recent progress and challenges, Bulletin of the American Meteorological Society, 95(3), 409-426, https://doi.org/10.1175/BAMS-D-11-00263.1, 2014.

285 Sutskever, I., Vinyals, O., and Le, Q. V.: Sequence to Sequence Learning with Neural Networks, in: Advances in Neural Information Processing Systems27, edited by Ghahramani, Z., Welling, M., Cortes, C., Lawrence, N. D., and Weinberger, K. Q., pages 3014-3112, in: NIPS, https://arxiv.org/abs/1409.3215v3, 2014.

Tian, L., Xutao, L., Yunming, Y., Pengfei, X., Yan, L.: A Generative Adversarial Gated Recurrent Unit Model for Precipitation Nowcasting, IEEE Geoscience and Remote Sensing Letters, 17(4),601-605, 2020.

290 Wang, G., Liu, L., Ruan, Z.: Application of Doppler Radar Data to Nowcasting of Heavy Rainfall, Journal of Applied Meteorological Science, 388-395+417, 2007.

Wang, Z., Bovik, A.C., Sheikh, H.R., Simoncelli, EP.: Image quality assessment: from error visibility to structural similarity, IEEE transactions on image processing, 13(4), 600-612, 2004.

Xie, P., Li, X., Ji, X., Chen, X., Chen, Y., Jia, L., Ye, Y.: An Energy-Based Generative Adversarial Forecaster for Radar Echo

Map Extrapolation, IEEE Geoscience and Remote Sensing Letters, PP(99):1-5, 2020. 